\title{
A pre-clinical model combining cryopreservation technique with precision-cut slice culture method to assess the in vitro drug response of hepatocellular carcinoma
}

\author{
YUAN ZHANG $^{1-3}$, ZHEN-YU WANG $^{1,4}$, HONG-SHU JING $^{1,4}$, HONG-DAN ZHANG $^{5}$, \\ HE-XIN YAN ${ }^{1,6}$, JIAN-XIA FAN ${ }^{2,3^{*}}$ and BO ZHAI ${ }^{1,4^{*}}$ \\ ${ }^{1}$ Department of Interventional Oncology, Renji Hospital, School of Medicine, Shanghai Jiao Tong University, \\ Shanghai 200127; ${ }^{2}$ International Peace Maternity and Child Health Hospital, School of Medicine, \\ Shanghai Jiao Tong University; ${ }^{3}$ Shanghai Key Laboratory of Embryo Original Diseases, Shanghai 200030; \\ ${ }^{4}$ State Key Laboratory of Oncogenes and Related Genes, Shanghai Cancer Institute, Renji Hospital, School of Medicine, \\ Shanghai Jiao Tong University, Shanghai 200127; ${ }^{5}$ Shanghai Celliver Biotechnology Co. Ltd., Shanghai 200120; \\ ${ }^{6}$ Department of Anesthesiology and Critical Care Medicine, Renji Hospital, School of Medicine, \\ Shanghai Jiao Tong University, Shanghai 200127, P.R. China
}

Received October 14, 2021; Accepted January 28, 2022

DOI: $10.3892 /$ ijmm.2022.5107

\begin{abstract}
Models considering hepatocellular carcinoma (HCC) complexity cannot be accurately replicated in routine cell lines or animal models. We aimed to evaluate the practicality of tissue slice culture by combining it with a cryopreservation technique. We prepared 0.3 -mm-thick tissue slices by a microtome and maintained their cell viability using a cryopreservation technique. Slices were cultured individually in the presence or absence of regorafenib (REG) for $72 \mathrm{~h}$. Alterations in morphology and gene expression were assessed by histological and genetic analysis. Overall viability was also analyzed in tissue slices by CCK-8 quantification assay and fluorescent staining. Tissue morphology and cell viability were evaluated to quantify drug effects. Histological and genetic analyses showed
\end{abstract}

Correspondence to: Dr Bo Zhai, Department of Interventional Oncology, Renji Hospital, School of Medicine, Shanghai Jiao Tong University, 160 Pujian Road, Shanghai 200127, P.R. China

E-mail: zhaiboshi@sina.com

Dr Jian-Xia Fan, International Peace Maternity and Child Health Hospital, School of Medicine, Shanghai Jiao Tong University, 910 Hengshan Road, Shanghai 200030, P.R. China

E-mail: fanjianxia122@126.com

${ }^{*}$ Contributed equally

Abbreviations: HCC, hepatocellular carcinoma; H\&E, hematoxylin and eosin; IHC, immunohistochemistry; LDH, lactate dehydrogenase; REG, regorafenib; CCK-8, Cell Counting Kit-8

Key words: pre-clinical model, cryopreservation, slice culture, drug response, hepatocellular carcinoma that no significant alterations in morphology and gene expression were induced by the vitrification-based cryopreservation method. The viability of warmed HCC tissues was up to $90 \%$ of the fresh tissues. The viability and proliferation could be retained for at least four days in the filter culture system. The positive drug responses in precision-cut slice culture in vitro were evaluated by tissue morphology and cell viability. In summary, the successful application of precision-cut HCC slice culture combined with a cryopreservation technique in a systematic drug screening demonstrates the feasibility and utility of slice culture method for assessing drug response.

\section{Introduction}

Liver cancer is one of the most commonly diagnosed types of malignant cancer and there are approximately 850,000 new cases diagnosed yearly worldwide (1). The high incidence of HCC has induced the development of novel targeted and personalized therapies (2). Personalization of cancer treatment requires the reliable prediction of chemotherapy responses in individual patients. Various strategies have been applied to generate primary cultures from individual tumors which include 2D cell culture of dissociated tumor cells, 3-D spheroid cultures and patient-derived mouse xenograft cultures (3-7). However, the difficulties in replicating the heterogeneous microenvironment in the primary tumor reduce their efficiency in drug experiments (8). It was estimated that over $90 \%$ of novel anticancer drugs fail in clinical trials because these models could not simulate complete tissue structure and maintain the biological heterogenicity of the primary tumor (9). For these reasons, it is crucial for us to create novel models that are more predictive of in vivo efficacy.

Precision-cut slice is a new method of tissue culture in vitro, which is derived directly from the primary tumor (10). However, there is no preservation method applied to maintain 
living fresh tissue. Conventional preservation of fresh tumor tissue such as formalin-fixed paraffin-embedded samples and flash freezing in liquid nitrogen always leads to the absolute inactivation of the fresh tissue. Therefore, a reliable and efficient cryopreservation method for living tissue is indispensable. Vitrification-based cryopreservation method can be developed to preserve fresh tissue, by which the biological characteristics of the original tumor can be retained and the utilization of specimens may be markedly improved (11).

In the present study, we explored a precision-cut slice culture method combined with a cryopreservation technique to establish a preclinical model, which is derived from fresh tissues of HCC patients. In addition, we demonstrated systematic optimization of HCC slices ex vivo by comparing different culture conditions. Moreover, this culture system allowed the detection of tumor responses to REG chemotherapy.

\section{Materials and methods}

Collection of HCC specimens. From October, 2019 to February, 2020, surgically resected specimens were obtained from 30 HCC patients at the Renji Hospital Affiliated to Shanghai Jiao Tong University School of Medicine (Shanghai, China). Samples were maintained at $4^{\circ} \mathrm{C}$ on ice and transported in preservation medium (Tissue Mate ${ }^{\mathrm{TM}}$; Celliver Biotechnology Co. Ltd.). Details are illustrated in Fig. 1. This investigation was approved by the Ethics Committee of Renji Hospital and followed the guidelines of The Declaration of Helsinki. All patients provided written informed consent. The inclusion criteria were: i) pathological diagnosis of HCC; ii) no patients had received any prior treatment; iii) the maximum diameter of a single tumor was more than $2 \mathrm{~cm}$; iv) Child-Pugh score A or B. The exclusion criteria were: i) Child-Pugh score C; ii) exceptional circumstances, such as syphilis and acquired immune deficiency syndrome.

Cryopreservation and warming procedures. All specimens were cut into $1-\mathrm{mm}$-thick slices in a metal mold before cryopreservation (Fig. 2C). Cryopreservation solutions (LT2601; Tissue Mate ${ }^{\mathrm{TM}}$ ) and warming solutions (LT2602; Tissue Mate $^{\mathrm{TM}}$ ) were provided by Celliver Biotechnology Co. Ltd. (Fig. 2B). For tissue cryopreservation, vitrification solution 1 (V1), vitrification solution 2 (V2) and vitrification solution 3 (V3) were pre-warmed in a $2 \sim 8^{\circ} \mathrm{C}$ water bath. Fresh HCC tissues were cleaned twice with sterile PBS and trans-

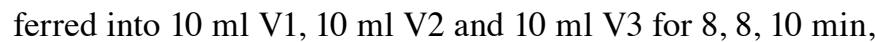
respectively. Tissues were then placed onto a thin metal strip and submerged into liquid nitrogen for at least $5 \mathrm{~min}$. Finally, the strips with tissue were placed into frozen storage tubes and preserved in the nitrogen canister. The tissue samples were stored in the liquid nitrogen. For tissue warming, the frozen storage tubes were removed from the nitrogen canister and the strips with the cryopreserved biopsy tissues were quickly transferred into $30 \mathrm{ml}$ warming solution 1 (T1), and incubated for $3 \mathrm{~min}$ in a $37^{\circ} \mathrm{C}$ water bath. The tissues were then transferred into $10 \mathrm{ml}$ warming solution 2 (T2) and $10 \mathrm{ml}$ warming solution 3 (T3) for 5 and $10 \mathrm{~min}$, respectively, at room temperature. Warmed tissues were cleaned twice with sterile PBS and kept on ice (Fig. 2D). The timeline of cryopreservation and warming procedures are depicted in Fig. 2A.
Tissue slice preparation and cultivation. Surgically resected specimens were cut into $300-\mu \mathrm{m}$-thick precision-cut slices using a microtome for slice preparation (Bio-Gene Technology, Ltd.) (Fig. 2E). A thickness of $300 \mu \mathrm{m}$ was considered the most suitable thickness for HCC after several early slicing pre-experiments (Fig. 2F). Parameter settings, such as the frequency and amplitude of vibration slicing, were determined by the diverse cirrhosis degree and tumor stage. Tissue slices (diameter, $2 \mathrm{~mm}$ ) were then prepared using a hand-held coring tool (Fig. 2G), and all the procedures were performed under sterile conditions. One-third of the precision-cut slices were maintained on Transwell inserts (pore size, $0.4 \mu \mathrm{m}$; Corning, Inc.) (Fig. 2I). One-third of the precision-cut slices were individually submerged in medium (Fig. 2H) and incubation was performed on a shaking platform (TYZD-III, QiQian Technology, Ltd.). The remaining precision-cut slices were cultured statically in medium as control (Fig. 2H). Cultivation was performed in 12-well plates containing $450 \mu \mathrm{l}$ DMEM medium $\left(\mathrm{Gibco}^{\mathrm{TM}}\right.$; Thermo Fisher Scientific, Inc.) with $10 \%$ fetal bovine serum $\left(\mathrm{Gibco}^{\mathrm{TM}}\right.$; Thermo Fisher Scientific, Inc.), penicillin and streptomycin (100 U/ml; Gibco ${ }^{\mathrm{TM}}$; Thermo Fisher Scientific, Inc.), and kept at $37^{\circ} \mathrm{C}$ in a humidified incubator with $5 \% \mathrm{CO}_{2}$.

Cell Counting Kit-8 (CCK-8) assay. A CCK-8 assay (Dojindo Molecular Technologies, Inc.) was used to evaluate the viability of tissue slices at each time point (24, 48, 72, $96 \mathrm{~h})$. DMEM (90 $\mu \mathrm{l} /$ well) and CCK-8 solution (10 $\mu \mathrm{l} /$ well) were added into 96 -well plates. The tissue slices were added one slice/well. The plates were maintained at $37^{\circ} \mathrm{C}$ in a humidified incubator with $5 \% \mathrm{CO}_{2}$ for $2 \mathrm{~h}$. The slices were removed from the 96-well plates and the plates were transferred to microplate reader (Multiskan GO; Thermo Fisher Scientific, Inc.). The absorbance at $450 \mathrm{~nm}$ was measured and three wells were tested for each sample at each time point.

Calcein-AM cell viability assay and Hoechst 33342 staining. The Live/Dead ${ }^{\circledR}$ Viability Assay kit (Nanjing KeyGen Biotech Co.) and Hoechst 33342 (Beyotime Institute of Biotechnology Co.) were stored at $-20^{\circ} \mathrm{C}$ and allowed to warm to room temperature prior to experimentation. The viability assay stock reagents (calcein-AM, $4 \mathrm{mM}$ ) were diluted to $1 \mu \mathrm{M}$ in physiological solution and mixed with $2 \mu \mathrm{g} / \mathrm{ml}$ Hoechst 33342 stock reagents at room temperature for $30 \mathrm{~min}$. Live cells are characterized by a bright green fluorescent and cell nucleus are blue. Representative images were captured with the Leica TCS SP8 confocal microscope (x20) (Leica Microsystems $\mathrm{GmbH})$. The ratio of living cells in the calcein-AM cell viability assay/Hoechst 33342 staining were calculated based on manual counting within 10 random microscopic fields.

Hematoxylin and eosin (H\&E)/immunohistochemical (IHC) staining. Tumor slices were formalin-fixed, embedded in paraffin and cut into $4-\mu \mathrm{m}$-thick sections. Paraffin sections $(4-\mu \mathrm{m})$ were stained with $\mathrm{H} \& \mathrm{E}$ at room temperature. IHC staining was carried out by standard protocols. Briefly, sections were de-waxed in xylene and rehydrated in graded ethanol, and heat-mediated antigen retrieval of tissue sections was carried out before being allowed to cool. Endogenous peroxidases were blocked using 0.9-3\% hydrogen peroxide for $10 \mathrm{~min}$, and non-specific antibody binding was blocked by 


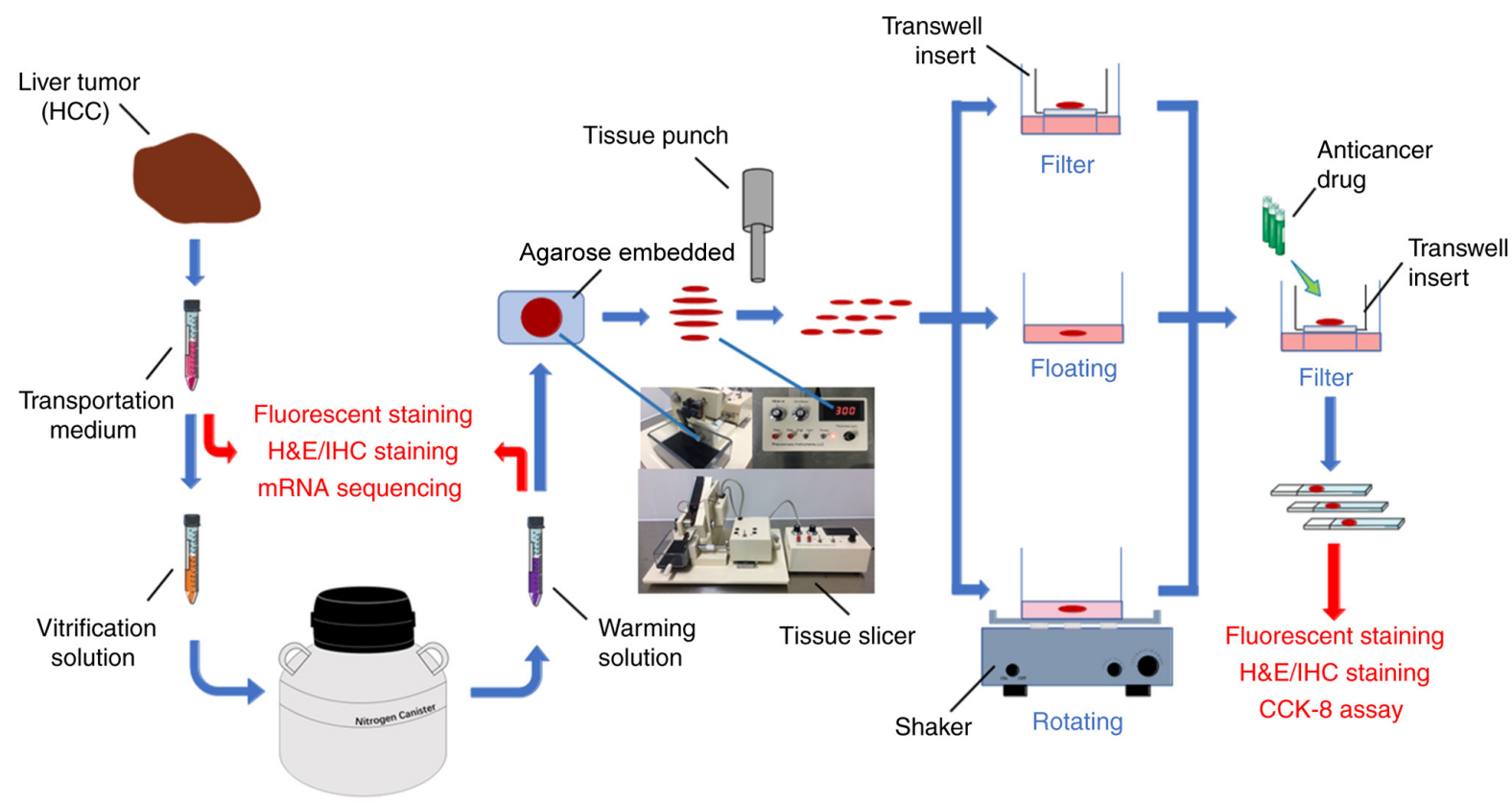

Figure 1. Workflow of the cryopreserved slice culture and drug testing. HCC tissues were obtained and transported to the laboratory in preservation medium at $4^{\circ} \mathrm{C}$ within $2 \mathrm{~h}$. Tissues were cryopreserved and stored in a nitrogen canister. Warmed tissues were used when required. The 1-mm-thick tissue slices were embedded in agarose and cut into 300- $\mu \mathrm{m}$-thick slices using a VF-300 microtome. Tissue slices $(2 \mathrm{~mm}$ in diameter) were maintained on different culture support. Slices were maintained on a Transwell insert and treated with REG at concentration of $20 \mu \mathrm{M}$. Experiments were performed to evaluate the efficacy of cryopreservation combined with slice cultivation in the assessment of anticancer drug responses by viability assay and fluorescent staining. HCC, hepatocellular carcinoma; REG, regorafenib; H\&E, hematoxylin and eosin; IHC, immunohistochemical.

A Cryopreservation procedure

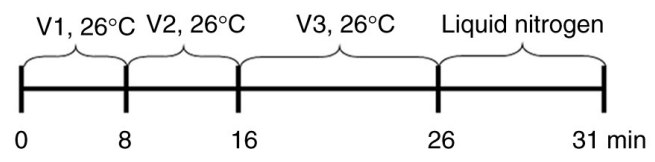

Warming procedure

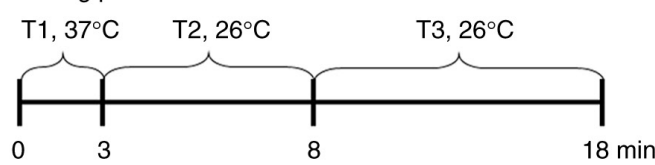

C

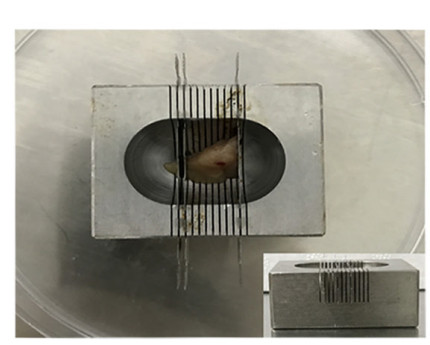

$\mathrm{D}$
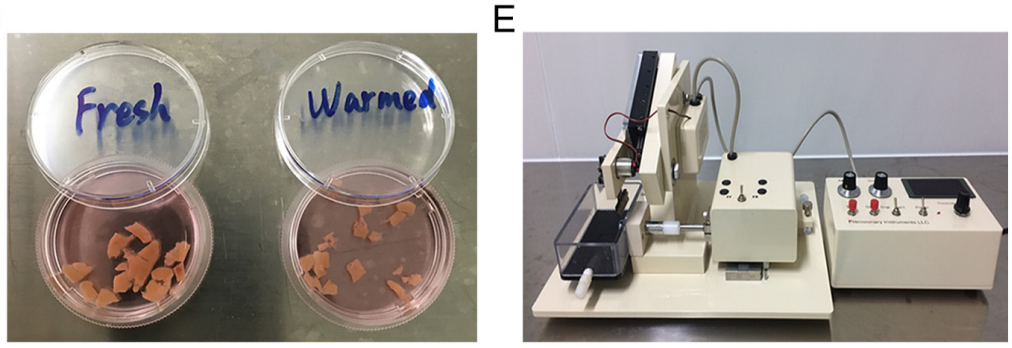

$\mathrm{F}$
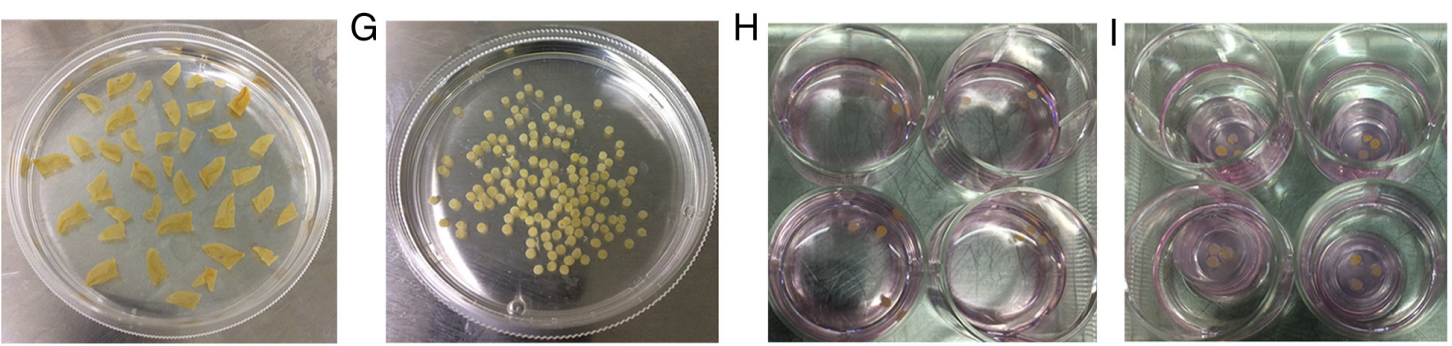

Figure 2. Cryopreservation technology and precision-cut tissue slice culture method. (A) The standardized time schedule of the cryopreservation and warming procedures. (B) Cryopreservation solutions and warming solutions. (C) Tissues were cut into 1-mm-thick slices by a stainless slicer in a metal mold. (D) Cryopreserved tissues and warmed tissues. (E) VF 300 microtome. (F) The 300- $\mu$ m-thick slices. (G) The 2-mm-diameter slices. (H) Floating culture. (I) Filter culture (Transwell insert). 
incubation with serum-free blocking solution or $10 \%$ normal serum block for $30 \mathrm{~min}$. Tissue sections were then incubated with the anti-Ki67 antibody (Ab15580; Abcam; 1:1,000 dilution), before being probed with the secondary antibodies Alexa Fluor ${ }^{\circledR} 488$ (Ab150077; Abcam; 1:1,000 dilution). Antibodies were visualized using 3,3'-diaminobenzidine chromogen and counterstained with Meyer's Hematoxylin for $2 \mathrm{~min}$. Sections were then dehydrated through graded alcohols, cleared in xylene and mounted. Confocal laser scanning microscopy (magnification, x20) was performed using an Olympus Corp. BX51 instrument. The ratio of proliferative cells in the Ki67 staining were calculated based on manual counting within 10 random microscopic fields.

Experimental methods for $m R N A$ sequencing. RNA purity was assessed using the kaiaoK5500 ${ }^{\circledR}$ Spectrophotometer (Beijing Kaiao Technology Development Co. Ltd.). RNA integrity and concentration were assessed using the RNA Nano 6000 Assay kit and the Bioanalyzer 2100 system (Agilent Technologies, Inc.). A total amount of $2 \mu \mathrm{g}$ RNA/sample was used as input material for the RNA sample preparations. Sequencing libraries were generated using NEBNext ${ }^{\circledR}$ Ultra $^{\text {TM }}$ RNA Library Prep kit for Illumina ${ }^{\circledR}$ (E7530L; New England BioLabs, Inc.), following the manufacturer's recommendations, and index codes were added to attribute sequences to each sample. Briefly, mRNA was purified from the total RNA using poly-T oligo-attached magnetic beads. Fragmentation was carried out using divalent cations under elevated temperature in NEBNext ${ }^{\circledR}$ First Strand Synthesis Reaction Buffer (5X) (New England BioLabs, Inc.). First-strand cDNA was synthesized using random hexamer primer and RNase H. Second-strand cDNA synthesis was subsequently performed using buffer, dNTPs, DNA polymerase I and RNase H. The library fragments were purified with QiaQuick PCR kits (Qiagen, Inc.) and elution with EB buffer, then terminal repair, A-tailing and adapter adding were implemented. The products were retrieved and PCR was performed, and then the library was completed. The RNA concentration of the library was measured using a Qubit ${ }^{\circledR}$ RNA Assay kit in Qubit ${ }^{\circledR} 3.0$ (Thermo Fisher Scientific, Inc.) for preliminary quantification, and then diluted to $1 \mathrm{ng} / \mu \mathrm{l}$. Insert size was assessed using the Agilent Bioanalyzer 2100 system (Agilent Technologies, Inc.), and qualified insert size was accurately quantified using the StepOnePlus ${ }^{\mathrm{TM}}$ Real-Time PCR System (Thermo Fisher Scientific, Inc.; library valid concentration, $>10 \mathrm{nM}$ ). The clustering of the index-coded samples was performed on a cBot cluster generation system using a HiSeq PE Cluster kit v4-cBot-HS (Illumina, Inc.) according to the manufacturer's instructions. After cluster generation, the libraries were sequenced on an Illumina, Inc. platform and 150-bp paired-end reads were generated. The variations in gene expression were detected by different colors in the heat map.

Metabolic activity of $\mathrm{pH} / \mathrm{glucose} / \mathrm{LDH}$. For the testing of the potential of hydrogen $(\mathrm{pH})$, we extract $15 \mu \mathrm{l}$ culture medium from the slice culture system using a detecting instrument (InLab Ultra Micro-ISM, Mettler Toledo); Glucose was tested using a detecting instrument (GlucCell ${ }^{\mathrm{TM}}$, Brookfield), using $3 \mu \mathrm{l}$ of culture medium; For LDH (lactate dehydrogenase), a detection kit (G1780, Promega Corp.) in a 96-well plate was used. All the processes were conducted using the operation manuals provided by the suppliers.

Drug sensitivity test in vitro. Drug testing commenced after $24 \mathrm{~h}$ of slice culture and was performed for an additional $72 \mathrm{~h}$. For drug testing of slices in vitro, regorafenib (REG; MedChemExpress LLC) was used and tested at a concentration of 5,10 , and $20 \mu \mathrm{M}$, respectively. To investigate cell proliferation and tissue morphology, the slices were incubated with CCK-8 solution and stained with $\mathrm{H} \& \mathrm{E} /$ fluorescent dyes.

Statistical analysis. Statistical evaluations were performed using one-way ANOVA with Scheffe's post hoc tests by IBM SPSS Statistics 22.0 (IBM Corp). $\mathrm{P}<0.05$ was considered to indicate a statistically significant difference. Three repeats were performed.

\section{Results}

Biological characteristics of HCC tissues are maintained by vitrification-based cryopreservation and precision-cut slice method. All of the fresh HCC specimens were obtained from Renji Hospital Affiliated to Shanghai Jiao Tong University. The 30 patients included 23 men and 7 women with a mean age of 58 years. The workflow was strictly performed by standard procedures, as depicted in Fig. 1. The specific explanation is provided in the Materials and methods section. Human liver tissues were found to be very well sliceable and showed a good reproducibility as well as tissue viability. The 1-mm-thick HCC slices were cryopreserved and warmed according to the timeline in Fig. 2A, using cryopreservation solutions (Fig. 2B, left) and warming solutions (Fig. 2B, right). Thirty fresh specimens were derived from $30 \mathrm{HCC}$ patients. Half of each specimen was processed by cryopreservation and warming procedures, and the remaining tissues were used as the control group. The 300- $\mu \mathrm{m}$-thick precision-cut slices were made and cultured successfully (Fig. 2C-I). H\&E staining of fresh tissue slices revealed no obvious differences in the morphology when compared to the warmed tissue slices. From the fluorescent and IHC staining, we found that the living cell ratio was $93 \%$ in fresh tissues and $90 \%$ in warmed tissues, which indicated that no obvious difference was detectable between the fresh HCC and warmed HCC tissues (Fig. 3A and B). The heat map of the cancer-associated genes indicated that the color of the left column was mostly consistent with the right column (Fig. 3E). Only a small part of differential gene expression was detected from the volcano plot (Fig. 3C) and distribution of sample expression (Fig. 3D). According to the GO analysis, it was found that the differential genes were closely related to cell metabolism (Fig. 3F). Original data were uploaded to the Gene Expression Omnibus database (accession number GSE194095). Therefore, the variations in gene expression between fresh and warmed tissues were limited. These results confirmed that the vitrification-based cryopreservation method was able to largely maintain the biological activity and histological features of the HCC tissues.

Medium composition and culture mode are critical to tissue viability. In order to identify the best slice culture methods, we optimized the slicing process with different culture 
A
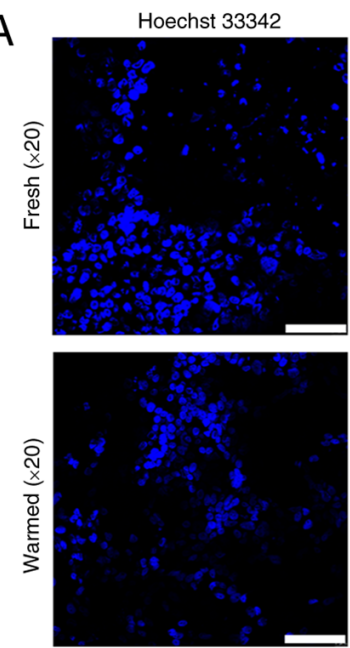

B

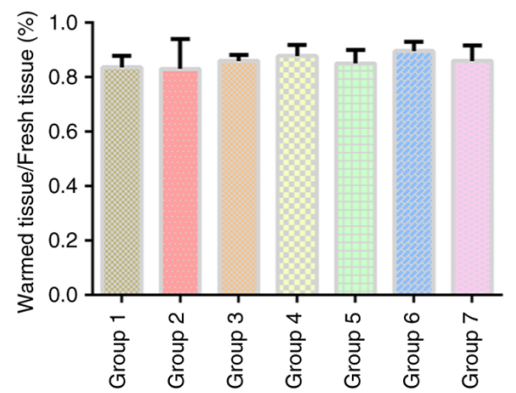

E
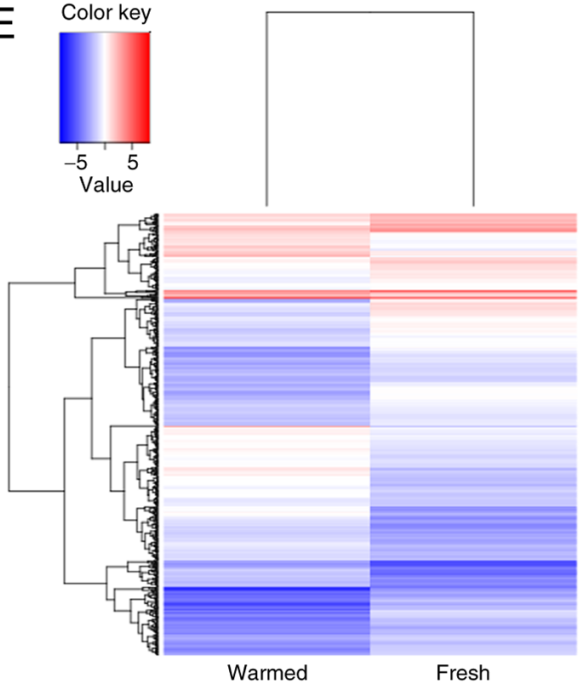

Calcenin-AM
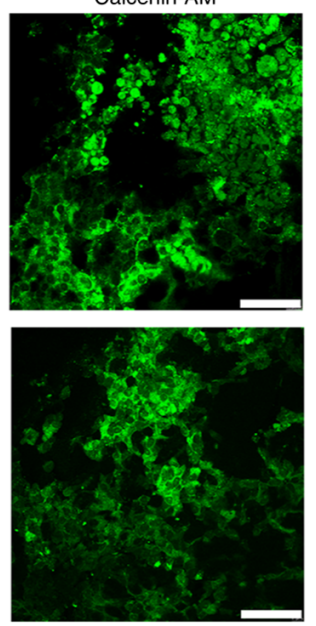
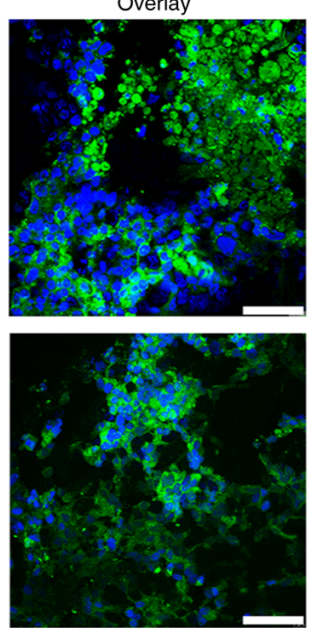

C

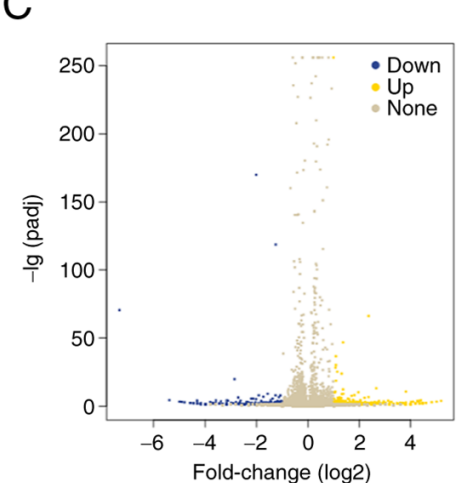

H\&E
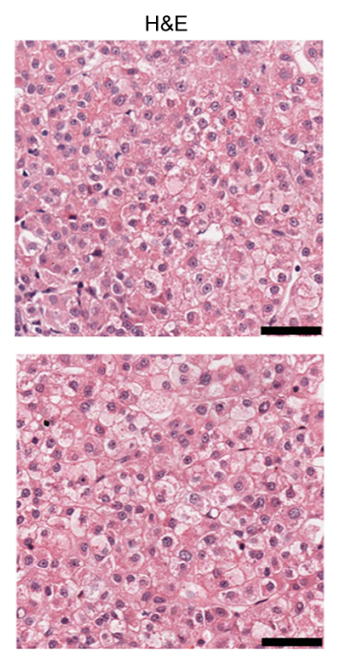
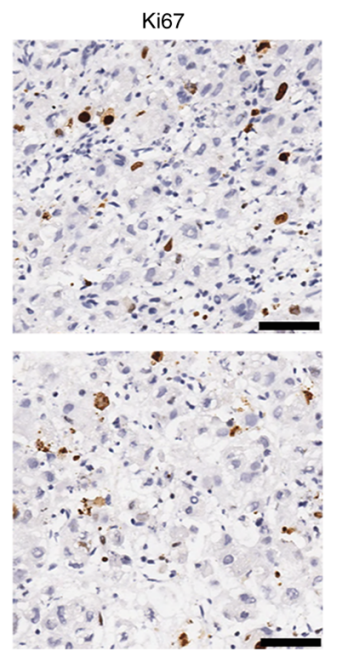

D

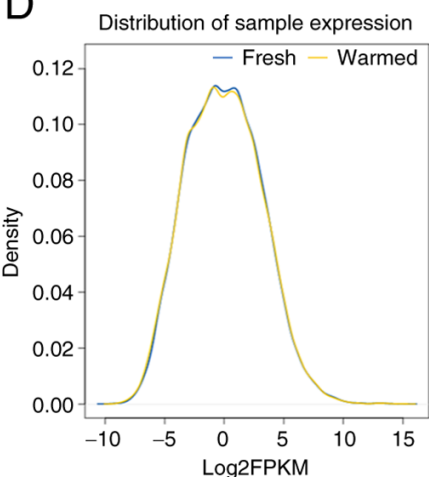

F

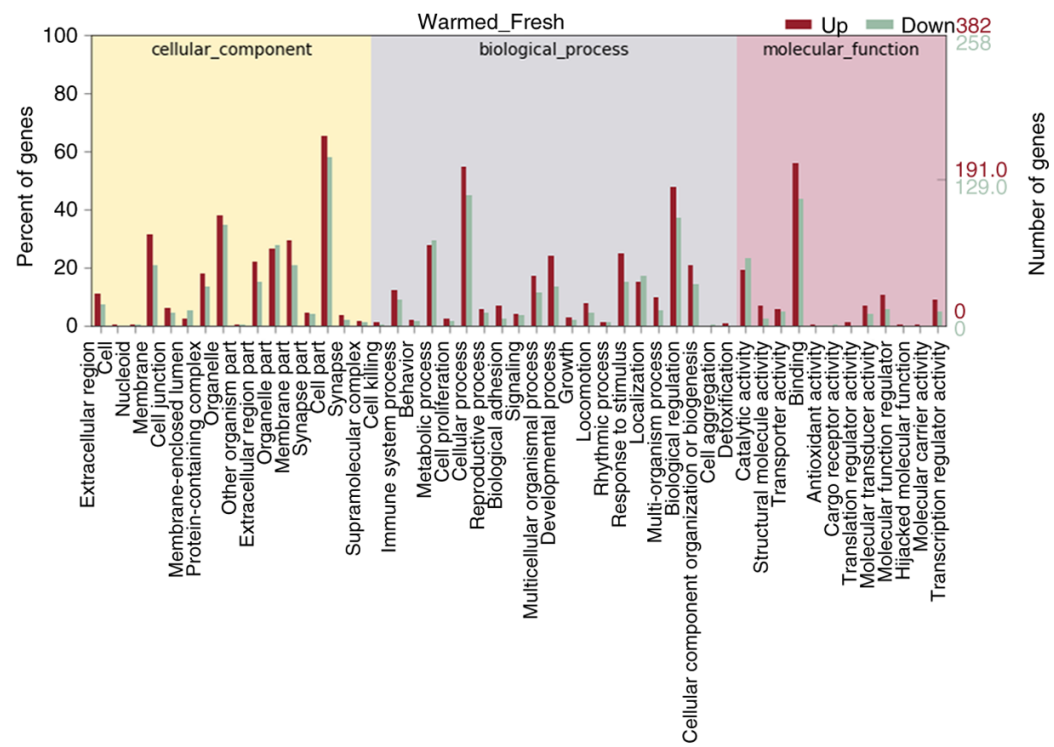

Figure 3. Effects of cryopreservation on the maintenance of the biological characteristics of tissues. (A) Calcein-AM/Hoechst staining and H\&E/IHC staining. Blue nuclei indicate both living and dead nuclei, while green sections represent the cytoplasm of living cells. IHC staining was used to indicate the percentage of cells expressing the proliferation marker Ki67. Scale bars, $50 \mu \mathrm{m}$. (B) The viability of fresh HCC slices was more than $10 \%$ of the warmed tissues, which was determined by CCK-8 assay ( $\mathrm{n}=7$ ). (C and D) Volcano plot and distribution of sample expression showed the differential gene expression between fresh and warmed tissues. (E) Heat map of mRNA sequencing. The color change in the heat map is defined as the difference in gene expression between fresh and warmed tissues. The deeper the red, the more greatly increased the gene expression. The deeper the blue, the lower the gene expression. (F) The Gene Ontology (GO) analysis indicated that differential genes were closely related to cell metabolism. HCC, hepatocellular carcinoma; H\&E, hematoxylin and eosin; IHC, immunohistochemistry.

methods and selected the optimal culture medium. Our results showed that Medium I (DMEM with high glucose $+10 \%$ FBS) could obviously maintain cell viability, especially from $48 \mathrm{~h}$ (Fig. 4A and B); Medium II (1640+10\% FBS) and Medium III (DMEM/F12) were not suitable for the slice culture (Fig. 4A). The filter cultures were viable for up to 

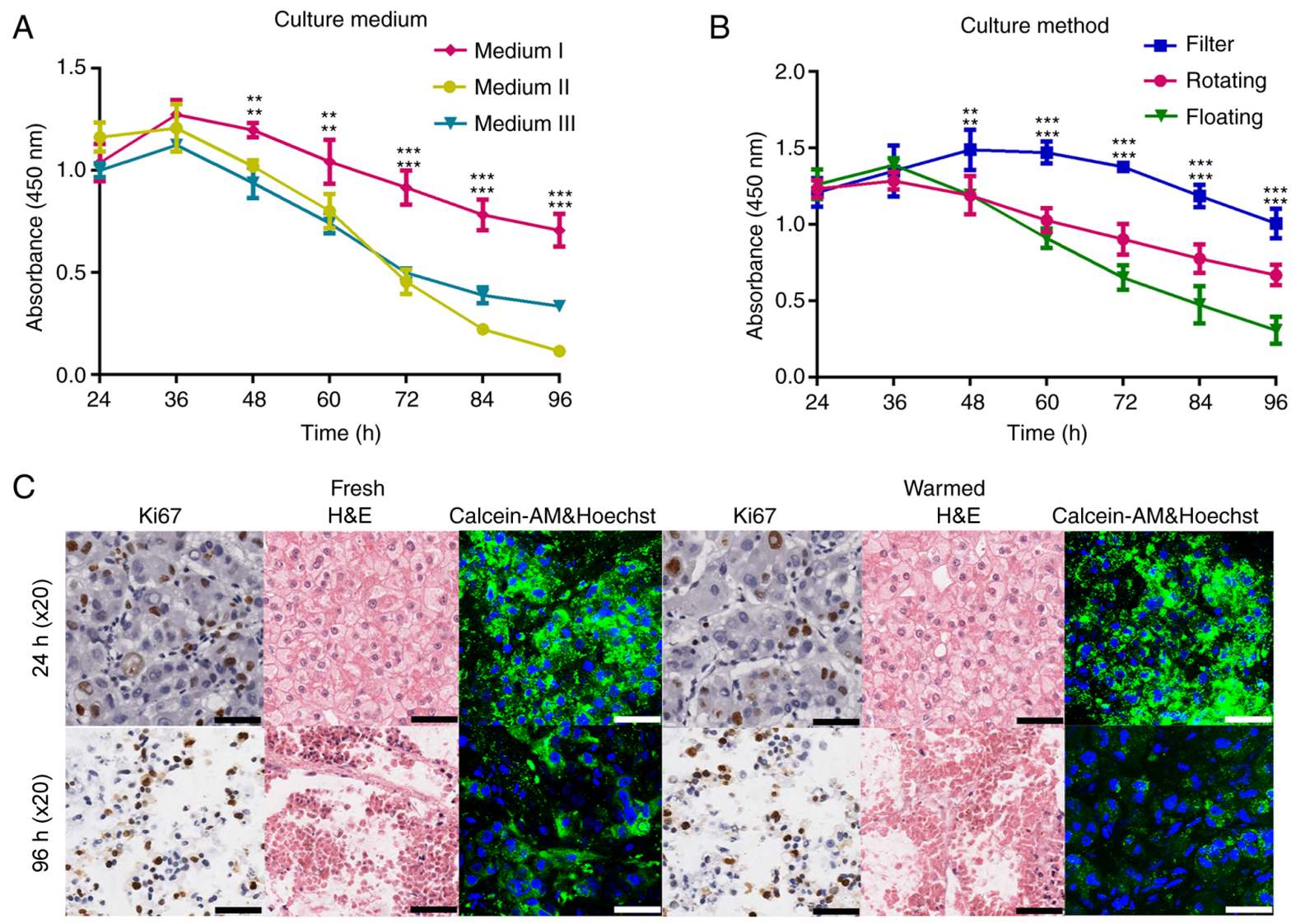

Fresh tissue

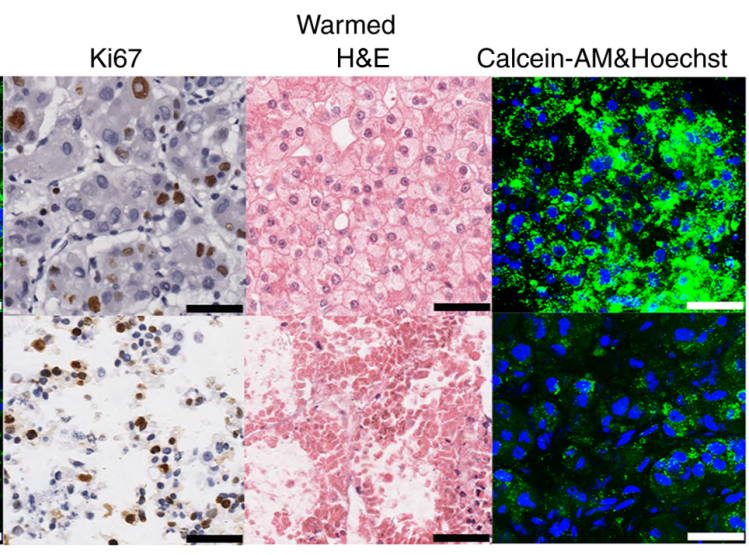

Warmed tissue
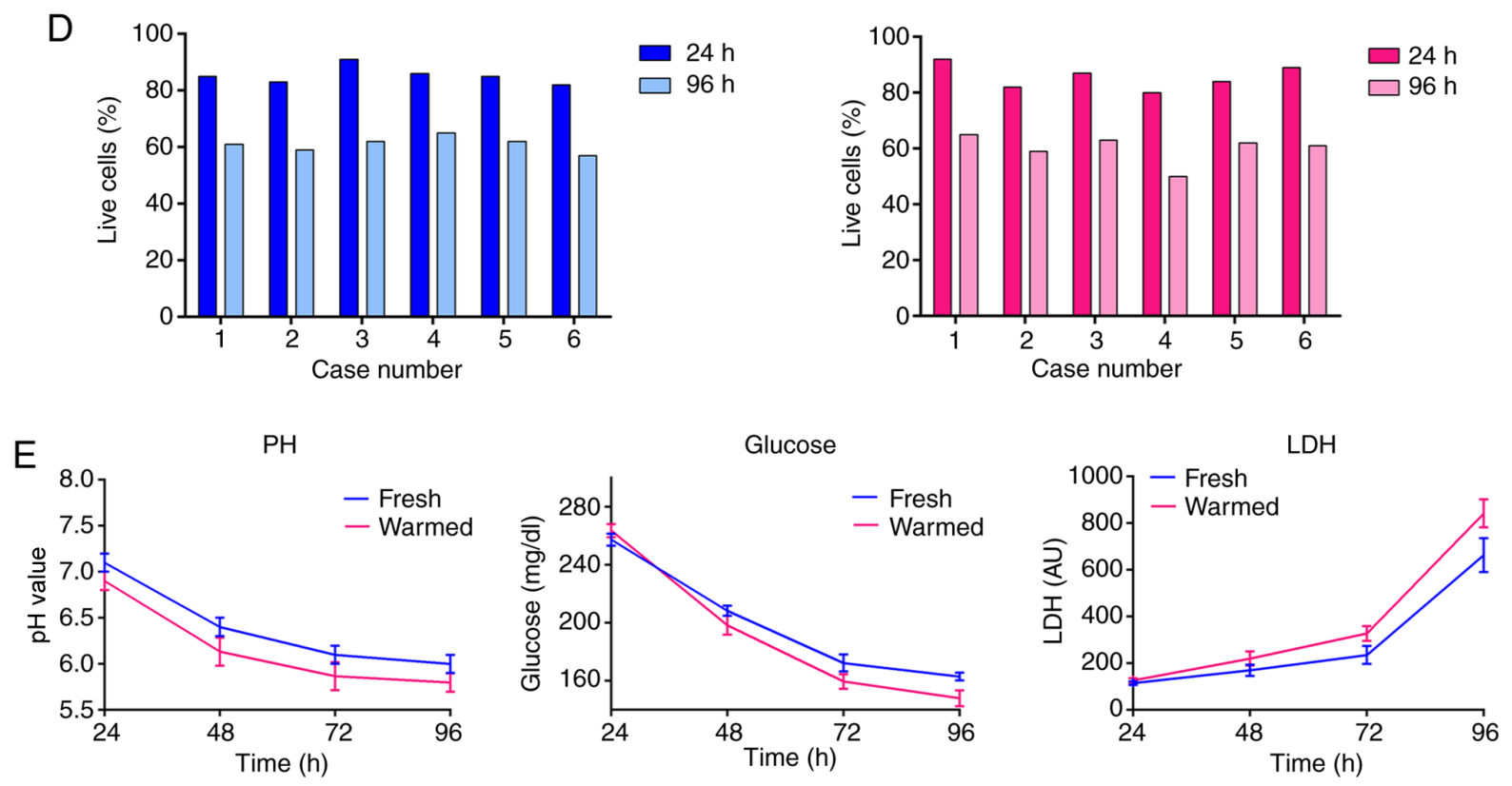

Figure 4. Optimization of medium composition and culture mode. (A) The adaption of Medium I (DMEM with high glucose $+10 \%$ FBS) was able to provide a higher cell viability and a longer culture time than Medium II $(1640+10 \%$ FBS $)$ or Medium III (DMEM/F12). ${ }^{* *} \mathrm{P}<0.01$ and ${ }^{* * * *} \mathrm{P}<0.001$. vs. Medium I. (B) Filter cultures were viable for up to 4 days and were able to achieve a higher cell viability than the floating and rotating culture. ${ }^{* *} \mathrm{P}<0.01$ and ${ }^{* * * *} \mathrm{P}<0.001$. vs. Filter. (C) Calcein-AM/Hoechst staining and H\&E/IHC staining. Scale bars, $50 \mu \mathrm{m}$. (D) The ratio of living cells in fresh and warmed slices after $96 \mathrm{~h}$. (E) The variation in $\mathrm{pH}$, glucose and LDH during the slice culture. H\&E, hematoxylin and eosin; IHC, immunohistochemistry; $\mathrm{pH}$, potential of hydrogen; LDH, lactate dehydrogenase.

4 days and could receive a higher cell viability than the floating and rotating cultures (Fig. 4B). Subsequently, we conducted slice culture on Transwell insert with DMEM combined with $10 \%$ FBS for $72 \mathrm{~h}$. As determined from the fluorescent and IHC staining, the living cell ratio was decreased slightly and a slight change in tissue morphological features was detected. In addition, these changes were observed both in fresh and warmed tissues (Fig. 4C and D). Moreover, we detected that the levels of $\mathrm{pH}$ and glucose were decreased while LDH was obviously increased (Fig. 4E). 
A

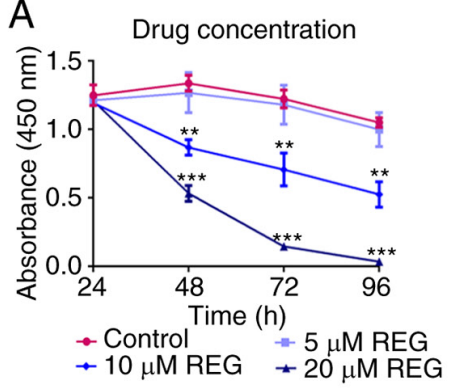

$\mathrm{B}$

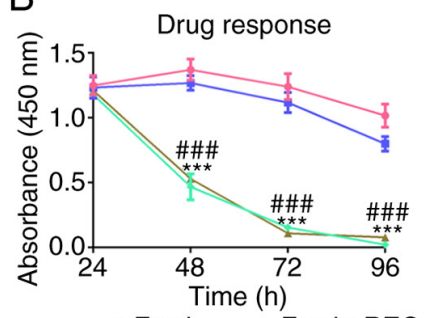

$\rightarrow$ Fresh $\rightarrow$ Fresh+REG
- Warmed
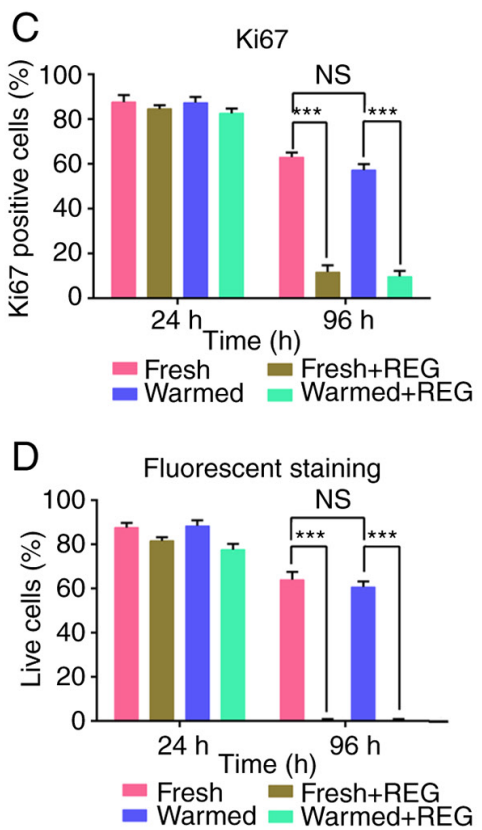

$\mathrm{E}$

E

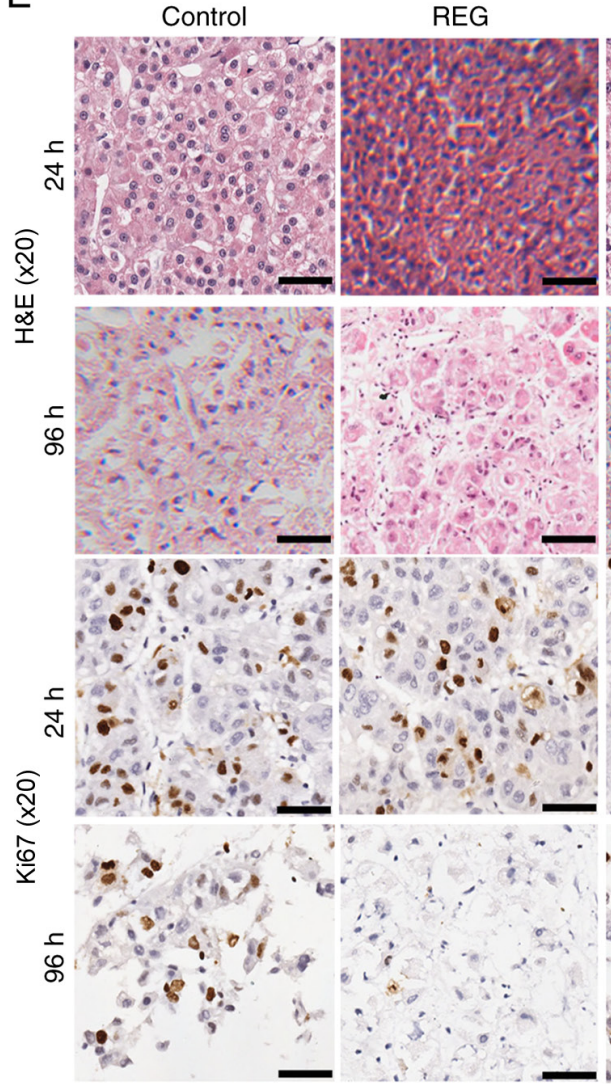

Fresh

REG
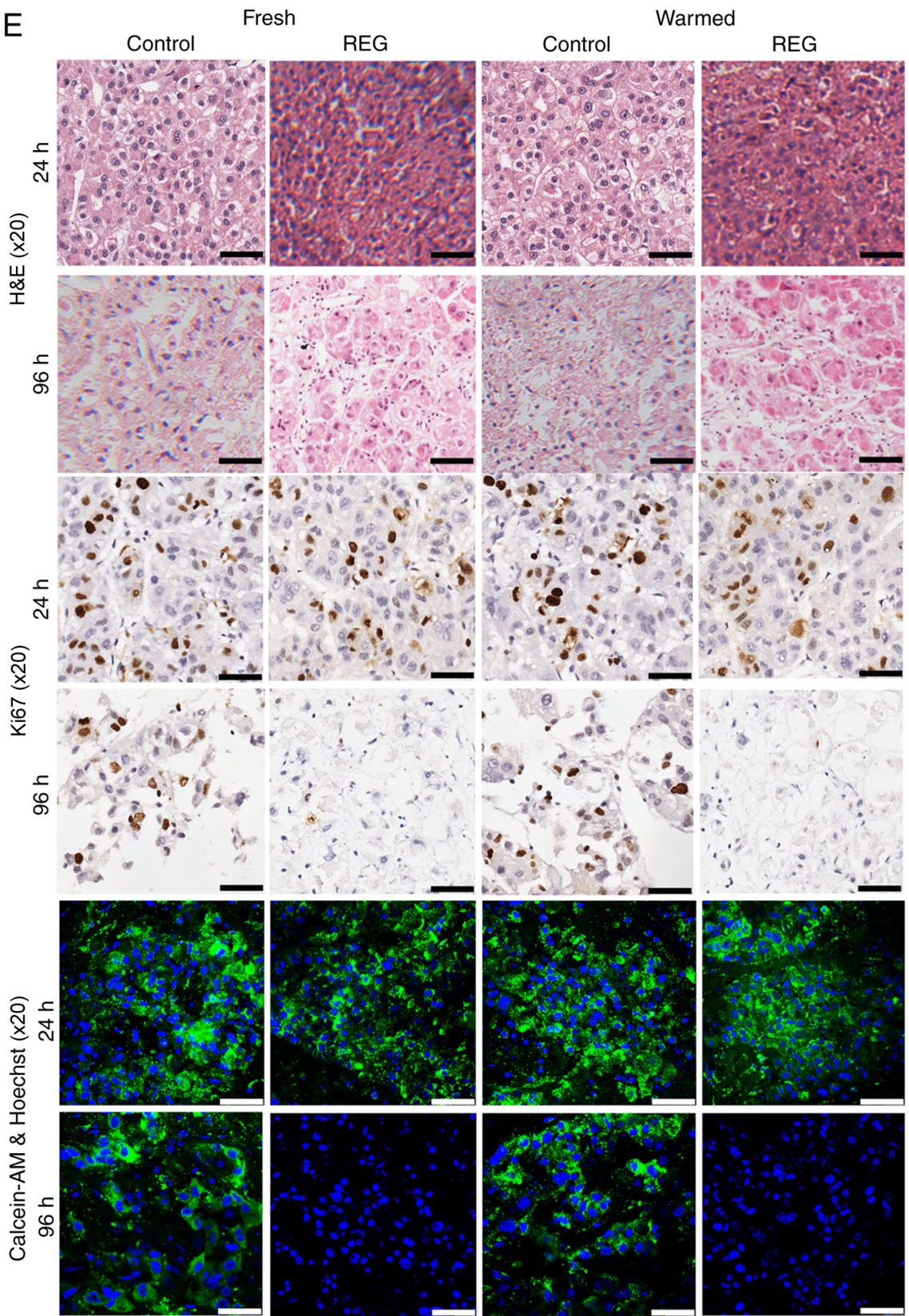

Figure 5. Positive drug responses in the slice culture model. (A) HCC slices were treated with different concentrations of REG $(5,10$, and $20 \mu \mathrm{M})$ for $72 \mathrm{~h}$. The control group was statistically different from the $10 \mu \mathrm{M}$ group and $20 \mu \mathrm{M}$ group, respectively. (B) CCK-8 cell viability assay was conducted for $72 \mathrm{~h} .{ }^{* * *} \mathrm{P}<0.01$

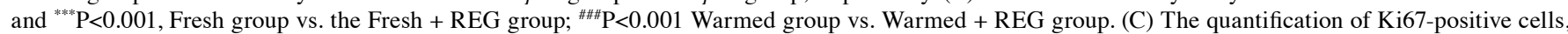
the proliferation rate before and after cryopreservation, was not statistically different as determined by Student's t-test. (D) The quantification of live cells, the number of live cells before and after cryopreservation, was not statistically different as determined by Student's t-test. ${ }^{* * *} \mathrm{P}<0.001$ and NS, not significant. The Fresh group was statistically different from the Fresh + REG group and the Warmed group was statistically different from the Warmed + REG group in 5B-D. (E) Morphological staining and fluorescent staining in fresh and warmed tissues during drug testing. Scale bars, $50 \mu \mathrm{m}$. HCC, hepatocellular carcinoma; REG, regorafenib.

Positive drug responses could be detected in slice culture model. Drug testing commenced after $24 \mathrm{~h}$ of slice culture and was performed for an additional $72 \mathrm{~h}$. To study the activity of anticancer drug REG in this tissue culture model, HCC slices were treated with different concentrations of REG $(5,10$ and $20 \mu \mathrm{M})$ for another $72 \mathrm{~h}$ as depicted in Fig. 5A. Our results revealed that $20 \mu \mathrm{M}$ was the most obvious concentration with which to significantly decrease the cell viability. Morphological staining and viability assays both indicated that no obvious differences were detectable after $24 \mathrm{~h}$ of slice culturing. However, compared with the control group, both fresh and warmed tissue slices in the drug treatment group cultured for $72 \mathrm{~h}$ showed a significant decrease in cell viability. In addition, tissue slices in the drug treatment group evidently lost the morphological structure of the original tumor (Fig. 5B-E).

\section{Discussion}

Research has indicated that a tissue slice culture system can be applied to perform preclinical and clinical studies for medical research (12-15). In our research, we describe precision-cut slice cultures as a novel model to perform ex vivo experiments on 
hepatocellular carcinoma (HCC) tumors, which preserves the three-dimensional structure of the tumor and provides an alternative to in vivo experiments. Our study was performed using standard procedures (Fig. 1). Studies have shown that there may be a drastic difference between a drug effect on cancer cells in a normal monolayer cell culture vs. a 3-D cell culture $(13,16,17)$. This evidence indicates the importance of normal tissue architecture and cell-cell communications that clearly exist in vivo.

One way to maintain these features is the tissue slice method, which was originally described for culture of breast and colon tumors (18-20). It has several important advantages. Firstly, the slice culture system provides the possibility to investigate the relationship between tumor cells and specific tumor microenvironments, which is suitable for the evaluation of drug effects and many other biological studies (21). Secondly, slice culture systems may reduce the need for animal testing, since they provide a biologically relevant platform for screening compounds. Normally, the exact control of thickness will be beneficial for full diffusion of nutrients and oxygen. The optimal thickness of slices was found to be related to the different type of tissue $(10,15,22)$. In order to determine the optimal thickness of slicing and culturing, we optimized the slicing process with a slicer and found that $300 \mu \mathrm{m}$ was the most suitable thickness for the HCC tumor slice after pre-experiments. Previous studies have reported that viability and proliferation could be retained for 3 to 7 days (10,23-25). Our results demonstrated that slices $(300-\mu \mathrm{m})$ cultured on filter inserts were viable for up to 4 days. We did not characterize later time points, but there were no significant signs of tissue deterioration after 4 days, suggesting that extended incubation may be possible if required for a specific functional assay.

In order to maintain the viability of the tissue and improve the utilization of specimens, a standardized vitrification-based cryopreservation method was developed. The cryopreservation and warming procedures should be implemented strictly in accordance with the time schedule. In fact, several types of cells, such as embryo and stem cells have been successfully vitrified $(26,27)$. The results of our research showed that no obvious difference was detected in the cell viability and morphological characteristics of the original tumor before and after cryopreservation. Gene expression analysis also showed that no significant alterations in gene expression were introduced by this cryopreservation method, except a slight alteration associated with cell metabolism. As determined by pre-experiments, no difference was induced by different lengths of preservation time in liquid nitrogen after cryopreservation. These findings further support the conclusion that vitrification is less damaging to cell viability and function due to the minimal ice crystallization in the process of cryopreservation (28).

To test and optimize the culture condition, the different composition of medium and the different growth support were compared. We adapted the culture medium for long-term expansion of the slice, because composition of the culture medium is highly important to maintain tumor slice viability. Similar as observed with other slices (24), the filter culture was superior to the rotating culture and floating culture. The reason may be attributed to a better oxygen supply of the tissue in the filter cultures. In the present research, tissue slices processed by a microtome all showed evident responses to anticancer drugs. The slice model therefore has tremendous potential in selecting sensitive anticancer drugs via examining the cell morphology and proliferation rate.

The present research demonstrated that HCC tissue slices could be effectively cryopreserved, and the tumor biological characteristics were well retained. The tissue slice model provides a better predictability of cancer drug response and improves the efficiency of precision or personalized treatment. Similar assays can be developed to investigate other drugs. At present, human tissue slice cultures have their limitations in regard to in vitro cultivation time and low throughput. Accordingly, further development is required to allow for high throughput analysis which is not possible in the current experiments. In addition, the cryopreserved method can also detect the toxicity of drugs to normal cells, which can be an area of future research.

\section{Acknowledgements}

Not applicable.

\section{Funding}

This study was supported by the National Natural Science Foundation of China (82070619).

\section{Availability of data and materials}

The datasets used and/or analyzed during the current study are available in the Gene Expression Omnibus database (accession number GSE194095).

\section{Authors' contributions}

YZ made substantial contributions to the conception and design of the study. ZYW made substantial contributions to the analysis and interpretation of the data. HSJ was involved in drafting the manuscript, and HDZ revised the draft critically for important intellectual content, and these authors also contributed to manuscript drafting and critical revisions on the intellectual content. HXY made substantial contributions to the conception and design of the study. JXF gave the final approval of the version to be published. YZ, JXF and BZ validated the data generated in this study. BZ agreed to be accountable for all aspects of the work in ensuring that questions related to the accuracy or integrity of any part of the work are appropriately investigated and resolved.

\section{Ethics approval and consent to participate}

Patient-derived specimens were used in the research. The manuscript does not contain experiments using animals. The investigation was approved (2019-09-15) by Ethics Committee of Renji Hospital, School of Medicine, Shanghai Jiao Tong University (Shanghai, China) and all patients provided written informed consent.

\section{Patient consent for publication}

Not applicable. 


\section{Competing interests}

The authors declare that they have no competing interests.

\section{References}

1. Wang J, Mao Y, Liu Y, Chen Z, Chen M, Lao X and Li S: Hepatocellular carcinoma in children and adolescents: Clinical characteristics and treatment. J Gastrointest Surg 21: 1128-1135, 2017.

2. Huang A, Yang XR, Chung WY, Dennison AR and Zhou J: Targeted therapy for hepatocellular carcinoma. Signal Transduct Target Ther 5: 146, 2020 .

3. Usui T, Sakurai M, Enjoji S, Kawasaki H, Umata K, Ohama T, Fujiwara N, Yabe R, Tsuji S, Yamawaki H, et al: establishment of a novel model for anticancer drug resistance in three-dimensional primary culture of tumor microenvironment. Stem Cells Int 2016: 7053872, 2016.

4. Yu Y, Wang Y, Xiao X, Cheng W, Hu L, Yao W, Qian Z and Wu W: MiR-204 inhibits hepatocellular cancer drug resistance and metastasis through targeting NUAK1. Biochem Cell Biol 97: 563-570, 2019.

5. Kumarasamy V, Vail P, Nambiar R, Witkiewicz AK and Knudsen ES: Functional determinants of cell cycle plasticity and sensitivity to CDK4/6 inhibition. Cancer Res 81: 1347-1360, 2021.

6. van de Wetering M, Francies HE, Francis JM, Bounova G, Iorio F, Pronk A, van Houdt W, van Gorp J, Taylor-Weiner A, Kester L, et al: Prospective derivation of a living organoid biobank of colorectal cancer patients. Cell 161: 933-945, 2015.

7. Bruna A, Rueda OM, Greenwood W, Batra AS, Callari M, Batra RN, Pogrebniak K, Sandoval J, Cassidy JW, Tufegdzic-Vidakovic A, et al: A biobank of breast cancer explants with preserved intra-tumor heterogeneity to screen anticancer compounds. Cell 167: 260-274. e222, 2016

8. Naipal KA, Verkaik NS, Sanchez H, van Deurzen CHM, den Bakker MA, Hoeijmakers JHJ, Kanaar R, Vreeswijk MPG, Jager A and van Gent DC: Tumor slice culture system to assess drug response of primary breast cancer. BMC Cancer 16: 78, 2016

9. Hickman JA, Graeser R, de Hoogt R, Vidic S, Brito C, Gutekunst $\mathrm{M}$ and van der Kuip H; IMI PREDECT Consortium: Three-dimensional models of cancer for pharmacology and cancer cell biology: Capturing tumor complexity in vitro/ex vivo. Biotechnol J 9: 1115-1128, 2014.

10. Holliday DL, Moss MA, Pollock S, Lane S, Shaaban AM, Millican-Slater R, Nash C, Hanby AM and Speirs V: The practicalities of using tissue slices as preclinical organotypic breast cancer models. J Clin Pathol 66: 253-255, 2013.

11. Zeng M, Yang QR, Fu GB, Zhang Y, Zhou X, Huang WJ, Zhang HD, Li WJ, Wang ZY, Yan HX and Zhai B: Maintaining viability and characteristics of cholangiocarcinoma tissue by vitrification-based cryopreservation. Cryobiology 78: 41-46, 2017.

12. Chadwick EJ, Yang DP, Filbin MG, Mazzola E, Sun Y, Behar O, Pazyra-Murphy MF, Goumnerova L, Ligon KL, Stiles CD and Segal RA: A brain tumor/organotypic slice co-culture system for studying tumor microenvironment and targeted drug therapies. $\mathrm{J}$ Vis Exp 105: e53304, 2015.

13. Kenny HA, Lal-Nag M, White EA, Shen M, Chiang CY, Mitra AK, Zhang Y, Curtis M, Schryver EM, Bettis S, et al: Quantitative high throughput screening using a primary human three-dimensional organotypic culture predicts in vivo efficacy. Nat Commun 6: 6220, 2015.
14. Parajuli N and Doppler W: Precision-cut slice cultures of tumors from MMTV-neu mice for the study of the ex vivo response to cytokines and cytotoxic drugs. In Vitro Cell Dev Biol Anim 45: 442-450, 2009

15. Gerlach MM, Merz F, Wichmann G, Kubick C, Wittekind C, Lordick F, Dietz A and Bechmann I: Slice cultures from head and neck squamous cell carcinoma: A novel test system for drug susceptibility and mechanisms of resistance. Br J Cancer 110: 479-488, 2014

16. Burdall SE, Hanby AM, Lansdown MR and Speirs V: Breast cancer cell lines: Friend or foe? Breast Cancer Res 5: 89-95, 2003.

17. Boj SF, Hwang CI, Baker LA, Chio IIC, Engle DD, Corbo V, Jager M, Ponz-Sarvise M, Tiriac H, Spector MS, et al: Organoid models of human and mouse ductal pancreatic cancer. Cell 160: 324-338, 2015.

18. van der Kuip H, Murdter TE, Sonnenberg M, McClellan M, Gutzeit S, Gerteis A, Simon W, Fritz P and Aulitzky WE: Short term culture of breast cancer tissues to study the activity of the anticancer drug taxol in an intact tumor environment. BMC Cancer 6: 86, 2006.

19. Schwerdtfeger LA, Nealon NJ, Ryan EP and Tobet SA: Human colon function ex vivo: Dependence on oxygen and sensitivity to antibiotic. PLoS One 14: e0217170, 2019.

20. Vaira V, Fedele G, Pyne S, Fasoli E, Zadra G, Bailey D, Snyder E, Faversani A, Coggi G, Flavin R, et al: Preclinical model of organotypic culture for pharmacodynamic profiling of human tumors. Proc Natl Acad Sci USA 107: 8352-8356, 2010.

21. Vesci L, Carollo V, Roscilli G, Aurisicchio L, Ferrara FF, Spagnoli L and Santis RD: Trastuzumab and docetaxel in a preclinical organotypic breast cancer model using tissue slices from mammary fat pad: Translational relevance. Oncol Rep 34: 1146-1152, 2015.

22. Grosso SH, Katayama ML, Roela RA, Nonogaki S, Soares FA, Brentani H, Lima L, Folgueira MAAK, Waitzberg AFL, Pasini FS, et al: Breast cancer tissue slices as a model for evaluation of response to rapamycin. Cell Tissue Res 352: 671-684, 2013.

23. Unger FT, Bentz S, Kruger J, Rosenbrock C, Schaller J, Pursche K, Spruessel A, Juhl H and David KA: Precision cut cancer tissue slices in anti-cancer drug testing. J Mol Pathophysiol 4: 108, 2015.

24. Davies EJ, Dong M, Gutekunst M, Närhi K, van Zoggel HJAA, Blom S, Nagaraj A, Metsalu T, Oswald E, Erkens-Schulze S, et al: Capturing complex tumour biology in vitro: Histological and molecular characterisation of precision cut slices. Sci Rep 5: 17187, 2015.

25. Maund SL, Nolley R and Peehl DM: Optimization and comprehensive characterization of a faithful tissue culture model of the benign and malignant human prostate. Lab Invest 94: 208-221, 2014.

26. Karimi-Busheri F, Rasouli-Nia A and Weinfeld M: Key issues related to cryopreservation and storage of stem cells and cancer stem cells: Protecting biological integrity. Adv Exp Med Biol 951: 1-12, 2016.

27. Ochota M, Wojtasik B and Niżański W: Survival rate after vitrification of various stages of cat embryos and blastocyst with and without artificially collapsed blastocoel cavity. Reprod Domest Anim 52 (Suppl 2): S281-S287, 2017

28. Elder E, Chen Z, Ensley A, Nerem R, Brockbank K and Song Y: Enhanced tissue strength in cryopreserved, collagen-based blood vessel constructs. Transplant Proc 37: 4625-4629, 2005.

This work is licensed under a Creative Commons Attribution-NonCommercial-NoDerivatives 4.0 International (CC BY-NC-ND 4.0) License. 